\title{
Numerical approach to simulating interference phenomena in a cavity with two oscillating mirrors
}

\author{
Paula I. Villar, ${ }^{1}$ Alejandro Soba, ${ }^{2}$ and Fernando C. Lombardo ${ }^{1}$ \\ ${ }^{1}$ Departamento de Física Juan José Giambiagi, FCEyN UBA and IFIBA CONICET-UBA, Facultad de Ciencias Exactas y Naturales, \\ Ciudad Universitaria, Pabellón I, 1428 Buenos Aires, Argentina \\ ${ }^{2}$ CNEA-CONICET Centro Atómico Constituyentes, Avenida General Paz, 1499, San Martín, Argentina
}

(Received 4 January 2017; published 15 March 2017)

\begin{abstract}
We study photon creation in a cavity with two perfectly conducting moving mirrors. We derive the dynamic equations of the modes and study different situations concerning various movements of the walls, such as translational or breathing modes. We can even apply our approach to one- or three-dimensional cavities and reobtain well-known results of cavities with one moving mirror. We compare the numerical results with analytical predictions and discuss the effects of the intermode coupling in detail as well as the nonperturbative regime. We also study the time evolution of the energy density and provide analytic justifications for the different results found numerically.
\end{abstract}

DOI: 10.1103/PhysRevA.95.032115

\section{INTRODUCTION}

The mechanical interaction between a moving mirror and the radiation field has always been an interesting issue of study. This is due to not only its practical purposes but also its representation of a fundamental system in quantum optics. Besides the change in the zero-point energy of the quantum vacuum provoked by static boundary conditions, there is a yet even more fascinating feature of the quantum vacuum that arises when considering dynamical boundary conditions. The presence of moving boundaries leads to a nonstable vacuum electromagnetic state, resulting in the generation of real photons, which is an amazing demonstration of the existence of quantum vacuum fluctuations of quantum electrodynamics (QED), referred to in the literature as the dynamical Casimir effect (DCE) [1] or motion-induced radiation. DCE is a common name ascribed to the processes in which photons are generated from vacuum due to the external time variation of boundary conditions for some field [2-4]. For the usual electromagnetic case this corresponds to the fast motion of a mirror or modulation of the dielectric properties of the mirror or intracavity medium [5].

Research in the field has mainly concentrated on onedimensional models, and a few works have studied more realistic three-dimensional models [6-10]. Since the amount of radiation generated is very small, much attention has been paid to the study of the one-dimensional model for which the effect is enhanced, for example, through the parametric resonance condition. The main difference between one- and three-dimensional cavities is that, while in one dimension the cavity's frequency spectrum is equidistant and leads to strong intermode interactions, in three dimensions the spectrum is, in general, nonequidistant and only a few modes may be coupled $[11,12]$. A cavity made of two perfectly parallel reflecting mirrors, one of which oscillates with a mechanical frequency equal to a multiple of the fundamental of the static cavity (while the other one is at rest), is a typical case in which the mentioned enhancement takes place. In most works this problem has been analytically studied through a perturbative expansion of the equations of motion of the field in terms of the small oscillation amplitude to find an approximative solution at short times. Although the direct measurement of radiation generated by moving mirrors is an important experimental challenge, it was asserted [13] that photon creation induced by timedependent boundary conditions has been observed experimentally in superconducting circuits. This experiment consists of a coplanar waveguide terminated by a superconducting quantum interference device, upon which a time-dependent magnetic flux is applied. A related experiment involving a Josephson metamaterial embedded in a microwave cavity has been described in Ref. [14]. These experiments stimulated new theoretical research on the role of dynamical Casimir physics in quantum information processing, quantum simulations, and engineering of nonclassical states of light and matter [15-19]. There are also ongoing experiments aimed at measuring the photon creation induced by the time-dependent conductivity of a semiconductor slab enclosed by an electromagnetic cavity [20], as well as proposals based on the use of high-frequency resonators to produce the photons and ultracold atoms to detect the created photons via superradiance [21].

Recent studies have indicated that DCE could even be implemented using a single two-level atom (qubit) with time-dependent parameters, such as the transition frequency or the atom-field coupling strength [22-26]. Generation of excitation from vacuum occurs due to the counterrotating terms in the Rabi Hamiltonian, which for many years had been neglected under the rotating-wave approximation. On the other hand, it has been proved that an ensemble of two-level atoms collectively coupled to the electromagnetic field of a cavity, driven at low frequencies and close to a quantum phase transition, stimulates the production of photons from the vacuum. This paves the way to an effective simulation of the DCE [27].

The case of cavities with two moving mirrors has also been considered via a perturbative treatment [28] and also using a technique inspired in the renormalization-group method, where the solution to the set of generalized Moore's equations is valid for both short and long times, improving perturbative approaches [29]. The authors considered two mirrors oscillating resonantly at the same frequency, allowing for different amplitudes and dephasing between mirrors in onedimensional cavities. As we shall see, the radiation induced strongly depends on the relation among the amplitudes, the frequency, and the different phases in the wall oscillations. 
As predicted in [29], we show that for some relations among the variables, there is constructive interference which leads to an exponential growth of particles inside the cavity. For some other relations, there is destructive interference and hence no vacuum radiation. We also show that our solution accounts for other physical solutions (the nonperturbative regime, for example) as for one oscillating wall.

In this paper we present a detailed numerical analysis of the particle creation rate, along with analytical considerations of the cases mentioned above. This paper is organized as follows. In Sec. II we present the equation of motion for the field modes of the electromagnetic field. In Sec. IV, we begin by analyzing the photon creation in the case where one wall of the cavity is at rest and the other one oscillates with a multiple frequency of the fundamental of the static cavity. Therein, we shall consider three-dimensional as well as one-dimensional cavities. In Sec. V, we concentrate on the case of two oscillating mirrors, one at each end of the cavity. We focus on the study of different cases of dephasing between the oscillating walls, i.e., zero- and $\pi$-dephasing movements. Finally, in Sec. VI we make our conclusions.

\section{BOUNDARY CONDITIONS}

We consider a rectangular cavity formed by perfectly conducting walls with dimension $L_{x}$ (also $L_{y}$ and $L_{z}$ if we consider a three-dimensional cavity). The mirrors placed at $x=L(t)$ and $x=R(t)$ are at rest for $t<0$ and begin to move at $t=0$ following a given trajectory $L(t)$ and $R(t)$, respectively. We assume these trajectories are as prescribed for the problem and that they work as a time-dependent boundary condition for the field.

We start with the field operator $A(x, t)$ for the vector potential which satisfies the wave equation $\square \vec{A}=0$. In terms of the creation $\left(a_{k}^{\dagger}\right)$ and annihilation $\left(a_{k}\right)$ operators, the field operator can be expressed as

$$
A(x, t)=\sum_{k}^{\infty}\left[\hat{a}_{k} \psi_{k}(x, t)+\hat{a}_{k}^{\dagger} \psi_{k}^{*}(x, t)\right] .
$$

In this equation, $\psi_{k}(x, t)$ are the mode functions of the field and are chosen to satisfy the boundary conditions, i.e., $\psi_{k}(L(t), t)=0$ and $\psi_{k}(R(t), t)=0$. We first consider the instantaneous mode basis for the $1+1$ field [30],

$$
\phi_{k}(x, t)=\sqrt{\frac{2}{R-L}} \sin \left[\frac{k \pi(x-L)}{R-L}\right],
$$

and write each mode as

$$
\psi_{k}(x, t)=\sum_{m=1}^{\infty} Q_{k m}(t) \phi_{m}(x, t),
$$

where $m$ is a positive integer.

By considering small-amplitude motions of the walls, we can write their trajectories as given by

$$
L(t)=A_{L} \epsilon_{L} \sin \left(\Omega_{L} t+\phi_{L}\right)
$$

and

$$
R(t)=L_{0}-A_{R} \epsilon_{R} \sin \left(\phi_{R}\right)+A_{R} \epsilon_{R} \sin \left(\Omega_{R} t+\phi_{R}\right),
$$

where $L_{0}$ is the cavity length in the static situation, $\epsilon_{R}$ and $\epsilon_{L}$ are small (dimensionless) parameters which characterize the small deviations of the walls from the initial static positions, and $A_{L}$ and $A_{R}$ are amplitudes. The mirrors can oscillate in phase or not, depending on the values of $\phi_{L}$ and $\phi_{R}$. Inserting the expansion of field modes into the wave equation and integrating over spatial dimensions lead to the equation of motion for the canonical variables, expressed as

$$
\ddot{Q}_{m}^{(n)}+\omega_{m}^{2}(t) Q_{m}^{(n)}=\frac{1}{L_{0}} \sum_{s} b_{m s} \dot{Q}_{s}^{(n)}+\frac{1}{4 L_{0}^{2}} \sum_{s}\left\{g_{m s}+\left[3(\dot{R}-\dot{L})^{2}+2 L_{0}(\ddot{R}-\ddot{L})\right] a_{m s}+\left(r_{m s}^{1}+r_{m s}^{2}\right)\right\} Q_{s}^{(n)}
$$

where, in the general $(3+1)$-dimensional case, $\omega_{m}(t)=\sqrt{\frac{(m \pi)^{2}}{[R(t)-L(t)]^{2}}+k_{\|}^{2}}$ is the mode frequency. The coefficients in the equation are defined as follows:

$$
\begin{aligned}
& a_{m s}= \begin{cases}1 & \text { if } m=s, \\
0 & \text { otherwise, }\end{cases} \\
& b_{m s}= \begin{cases}\frac{4 m s}{\left(m^{2}-s^{2}\right)}\left[\dot{R}(-1)^{m+s}-\dot{L}\right] & \text { if } m \neq s, \\
0 & \text { otherwise, }\end{cases} \\
& g_{m s}= \begin{cases}\frac{m s}{\left(m^{2}-s^{2}\right)}\left\{24 \dot{L}(\dot{R}-\dot{L})-24 \dot{R}(\dot{R}-\dot{L})(-1)^{m+s}+8 L_{0}\left[\ddot{R}(-1)^{m+s}-\ddot{L}\right]\right\} & \text { if } m \neq s, \\
3(\dot{L}-\dot{R})^{2}-2\left[L_{0}(\ddot{R}-\ddot{L})\right] & \text { if } m=s,\end{cases} \\
& r_{m s}^{1}= \begin{cases}\frac{16 m^{3} s}{\left(m^{2}-s^{2}\right)^{2}} \dot{L}(\dot{R}-\dot{L})\left[-1+(-1)^{m+s}\right] & \text { if } m \neq s, \\
2 m^{2} \pi^{2} \dot{L}(\ddot{R}+\ddot{L}) & \text { if } m=s,\end{cases} \\
& r_{m s}^{2}= \begin{cases}\frac{-16 m^{3} s}{\left(m^{2}-s^{2}\right)^{2}}(\dot{R}-\dot{L})\left[\dot{L}+(\dot{L}-2 \dot{R})(-1)^{m+s}\right] & \text { if } m \neq s, \\
\frac{2}{3}(\dot{R}-\dot{L})\left[3(\dot{L}-\dot{R})+m^{2} \pi^{2}(\dot{R}+\dot{L})\right] & \text { if } m=s .\end{cases}
\end{aligned}
$$




\section{NUMERICAL METHOD}

In this section we describe the numerical method used for solving the equation of motion of the field modes determined by Eq. (6). In order to solve the equation of motion of the $n$ modes, we perform a change of variables in order to obtain a new system of equations:

$$
\begin{aligned}
& \dot{Q}_{m}=U_{m}, \\
& \dot{U}_{m}=-\omega_{m}^{2}(t) Q_{m}+\sum_{s} S_{m s}(t) Q_{s},
\end{aligned}
$$

where $S_{m s}(t)$ is a bracket proportional to $Q_{s}$ in Eq. (6). We have dropped the supraindex for simplicity. The initial conditions, specified for each field mode in all cases, are

$$
Q_{k}^{(n)}(0)=\frac{1}{\sqrt{2 \omega_{n}}} \delta_{k, n}, \quad \dot{Q}_{k}^{(n)}(0)=-i \sqrt{\frac{\omega_{n}}{2}} \delta_{k, n},
$$

which indicate that the field modes and their derivatives are continuous at $t=0$, as long as $R(t), L(t), \dot{R}(t)$, and $\dot{L}(t)$ are smooth functions. For a time dependence of the boundary, either $R(t)$ or $L(t)$, which is not sufficiently smooth (for example, it has discontinuities in its time derivative), one may expect spurious particle creation.

We have used an integration scheme based on a fourth-order Runge-Kutta-Merson numerical method between $t=0$ and a maximum time $t_{\max }>0$. In all cases, the moving walls are at rest at $t=0$. and then the perturbation is turned on for times in the range $0<t<t_{F}$, with $t_{F}<t_{\max }$, where the walls remain static again (this can be applied to either one or two moving walls). For times $t<0$ and $t>t_{F}$, the cavity is a static one, and we know the set of orthonormal functions. The quantization of the system is straightforward through creation and annihilation operators:

$$
Q_{n}(t<0)=\frac{1}{\sqrt{2 \omega_{n}}}\left(\hat{a}_{n} e^{-i \omega_{n} t}+\hat{a}_{n}^{\dagger} e^{i \omega_{n} t}\right),
$$

with frequency $\omega_{n}(t)=1 / L_{0} \sqrt{(\pi n)^{2}+k_{\|}^{2}}$, where $L_{0}$ is the initial length of the cavity and $k_{\|}$is associated with the nondynamical dimensions of the cavity $\left(L_{y}\right.$ and $\left.L_{z}\right)$. The time-independent annihilation and creation operators $\hat{a}_{n}$ and $\hat{a}_{n}^{\dagger}$ associated with the particle notion for $t \leqslant 0$ are subject to the commutation relations $\left[\hat{a}_{n}, \hat{a}_{m}\right]=\left[\hat{a}_{n}^{\dagger}, \hat{a}_{m}^{\dagger}\right]=0$ and $\left[\hat{a}_{n}, \hat{a}_{m}^{\dagger}\right]=$ $\delta_{n m}$. The initial vacuum state $|0, t \leqslant 0\rangle$ is defined by

$$
a_{n}^{\dagger}|0, t \leqslant 0\rangle=0 \forall n .
$$

When the cavity dynamics is switched on at $t=0$ and the walls follow the prescribed trajectories $L(t)$ and $R(t)$, the field modes are coupled. Then, $Q_{n}$ can be written as

$$
Q_{n}(t \geqslant 0)=\sum_{m} \frac{1}{\sqrt{2 \omega_{m}}}\left[\hat{a}_{m} \epsilon_{n}^{m}(t)+\hat{a}_{m}^{\dagger} \epsilon_{n}^{*}(t)\right],
$$

with complex functions $\epsilon_{n}^{m}(t)$ that satisfy the equation of modes. When the motion ceases and the walls are at rest again for $t>t_{F}, Q_{n}(t)$ can be expressed again as

$$
Q_{n}\left(t \geqslant t_{F}\right)=\frac{1}{\sqrt{2 \omega_{n}^{1}}}\left(\hat{A}_{n} e^{-i \omega_{n}^{1}\left(t-t_{F}\right)}+\hat{A}_{n}^{\dagger} e^{i \omega_{n}^{1}\left(t-t_{F}\right)}\right),
$$

with $\omega_{n}^{1}=\omega_{n}^{1}\left(t \geqslant t_{F}\right)$ and the annihilation and creation operators $\hat{A}_{n}$ and $\hat{A}_{m}^{\dagger}$ corresponding to the particle notion for $t \geqslant t_{F}$. The final vacuum state $\left|0, t \geqslant t_{F}\right\rangle$ is defined by

$$
A_{n}^{\dagger}\left|0, t \leqslant t_{F}\right\rangle=0 \forall n \text {. }
$$

As expected, the initial-state particle operators $\hat{a}_{n}$ and $\hat{a}_{n}^{\dagger}$ are linked to the final-state operators $\hat{A}_{n}$ and $\hat{A}_{n}^{\dagger}$ by a Bogoliubov transformation $\hat{A}_{n}=\sum_{m}\left[A_{m n}\left(t_{F}\right) \hat{a}_{m}+B_{m n}^{*}\left(t_{F}\right) \hat{a}_{m}^{\dagger}\right]$. The total number of particles created in a mode $n$ during the motion of the wall is given by the expectation value of the particle number operator $\hat{A}_{n}^{\dagger} \hat{A}_{n}$ associated with the particle notion for $t \geqslant t_{F}$ with respect to the initial vacuum state:

$$
N_{n}\left(t_{F}\right)=\left\langle 0, t \leqslant\left|A_{n}^{\dagger} A_{n}^{\dagger}\right| 0, t \leqslant 0\right\rangle=\sum_{m}\left|B_{m n}\left(t_{F}\right)\right|^{2} .
$$

In order to obtain the numerical results presented in the following sections we proceed in the following way. Two cutoff parameters $\Lambda$ (for the field modes considered) and $\Lambda_{m}$ (for the number of canonical variables considered) are introduced to make the system of differential equations finite and suitable for a numerical treatment. The system of $n \times m$ coupled differential equations is then evolved numerically from $t=0$ up to a final time $t_{F}$, and the expectation value of Eq. (14) is calculated for several times in between. By doing so we interpret $t_{F}$ as a continuous variable such that Eq. (14) becomes a continuous function of time. Consequently, the stability of the numerical solutions with respect to the cutoffs has to be ensured. In particular $\Lambda$ will be chosen such that the numerical results for the number of particles created in single modes are stable. In most cases, it is enough to choose $\Lambda_{m}=\Lambda$. In our units, the spectral modes $k_{n}=\Omega_{n}$ are given in units of $1 / L_{0}$ ( $k_{n} L_{0}$ is dimensionless), and consequently, time is measured in units of $L_{0}$.

\section{ONE MOVING MIRROR}

We can start by studying photon creation when only one wall is moving following $R(t)$ and the other one is at rest in $x=0$ (for example, by setting $\epsilon_{L}=0$ and $\epsilon_{R} \neq 0$ ). In this case, we can take any value of $\phi_{R}$, say, $\phi_{R}=0$. If we excite the cavity with an external frequency $\Omega_{R}$ such that $\Omega_{R}=2 \omega_{1}$, we shall produce parametric resonance induced by the moving mirror at $x=R(t)$. We can consider either a one-dimensional cavity or a three-dimensional one since this approach can be applied to either $1+1$ or $3+1$ dimensions by taking into account that in the latter case, the "moving walls" are in the $x$ direction, while the field satisfies Dirichlet boundary conditions in the other walls of the cavity ( $y$ and $z$ directions). The important difference between one- and higher-dimensional cavities is that the frequency spectrum in only one spatial dimension is equidistant, while it is, in general, nonequidistant for more spatial dimensions. An equidistant spectrum yields strong intermode coupling, whereas in the case of a nonequidistant spectrum only a few or even more modes may be coupled, allowing for exponential photon creation in a resonantly vibrating three-dimensional cavity. For both Dirichlet boundary conditions, the eigenfrequencies inside the 
cavity satisfy the following condition:

$$
\omega_{n}=\frac{1}{L_{0}} \sqrt{(\pi n)^{2}+M^{2}},
$$

where $n$ is natural number and we have set $M^{2} \equiv k_{\|}^{2}$. If the field is massless (which corresponds to a one-dimensional cavity), then the spectrum is equidistant; that is, the difference between two consecutive eigenfrequencies is constant. Otherwise, if $M$ has arbitrary nonzero values, the spectrum is nonequidistant, corresponding to that of a three-dimensional cavity.

\section{Three-dimensional cavity}

As we stressed at the end of the previous section, when considering a three-dimensional cavity, the parallel component of the wave number $k_{\|}=\pi \sqrt{\left(n_{y} / L_{y}\right)^{2}+\left(n_{z} / L_{y}\right)^{2}}$ can be associated with the nondynamical cavity dimensions and can be identified with the "mass" of a massive field [10]. Consequently, the number of TE-mode photons created in a three-dimensional cavity equals the number of scalar particles of mass $k_{\|}$created in a one-dimensional cavity. Then, we perform the simulations by considering $\Omega=\Omega_{R}=2 \omega_{1}$ and different values of a mass $M$ for simulating the particle creation in a three-dimensional cavity. In Fig. 1, we can see the behavior of the $|B|^{2}$ coefficient of mode $n=1$ of the field for different values of $M$, say, $M=1, M=5$, and $M=10$. The particle creation is expected to be exponential as the frequency spectrum becomes more nonequidistant. This is achieved more clearly for bigger values of $M \gg \pi / L_{0}$. In a resonant vibrating cavity, the number of TE-mode photons created in the resonant mode $n$ increases exponentially in time as [10]

$$
N_{n}(t)=\sinh \left(\frac{n^{2} \pi^{2} \epsilon_{R} t}{2 \Omega L_{0}^{2}}\right)^{2} .
$$

We can see in Fig. 1 the expected exponential behavior as the mass increases. The gray solid curve (parallel to $M=10$ ) represents $N_{1}(t)$ as computed for $M=10$ in [10] using Eq. (16). We can also see that the behavior is similar, although in our cavity it is attenuated by the multiplicative factor included in the relationship between the number of created

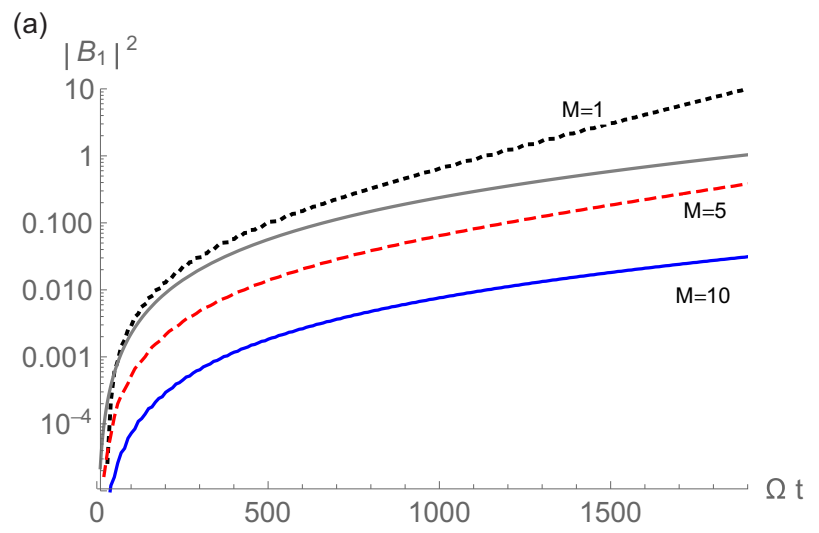

particles $N_{n}$ and the coefficient $\left|B_{n}\right|^{2}$. As we decrease the value of $M$, we reach a region of values where we can neglect the mass term inside the frequency definition and obtain an equidistant spectrum. In Fig. 1(b) we can see that the behavior induced by the resonant frequency $\Omega=2 \omega_{1}$ when $M \rightarrow 0$ changes considerably and cannot be fitted by Eq. (16) any longer.

\section{One-dimensional cavity}

When studying the case $M=0$, the numerical results should converge towards the well-known results for the massless case where all modes are coupled. In this case, we know that an eigenfrequency $k$ is defined by $\omega_{n}=\left(n \pi / L_{0}\right)$. Then, if we excite the system with $\Omega=2 \omega_{1}$, we should obtain a quadratic behavior for the field mode at times $\Omega t \ll 1 / \epsilon$. For a later time, we should expect a linear behavior. In Fig. 2, we present the behavior of $\left|B_{1}\right|^{2}$ for a value of $\epsilon=0.01$. For very short times, we can observe a quadratic behavior and then a linear one. However, for values bigger than $\Omega t \gg 1 / \epsilon$ we can see that the growth becomes exponential. The exponential behavior for longer times is equivalent to what has been found in Ref. [31]. The coupling to a large number of field modes derives in exponential growth at longer times in the very nonperturbative regime.

For an equidistant spectrum, we can see that the coupling between an infinite number of modes leads to quadratic growth in time of the number at short time scales $\left[\sim 1 /\left(\Omega \epsilon_{R}\right)\right]$ and a linear one in the long-time limit $\left[\sim 1 /\left(\Omega^{2} \epsilon_{R}^{2}\right)\right]$, as expected. In Figs. 2(a) and 2(b) we show the dependence upon $\epsilon$ of the quadratic and linear regimes. For example, for $\epsilon_{R}=0.001$, we can observe the same behavior but in a different temporal scale, as shown in Fig. 2(b). We can even compute the energy density for the case of a moving mirror for a one-dimensional field. In this case, the frequency spectrum is equidistant, and the energy grows quadratically for short times, as in Fig. 2. In Fig. 3, we show the energy behavior for $\epsilon_{R}=0.001$.

In order to show that the behavior at very long times is much enhanced by the finiteness of the number of modes, we show a comparison of different values of $\Lambda$ in Fig. 4. Therein, we show with the solid blue line the simulation for a cavity

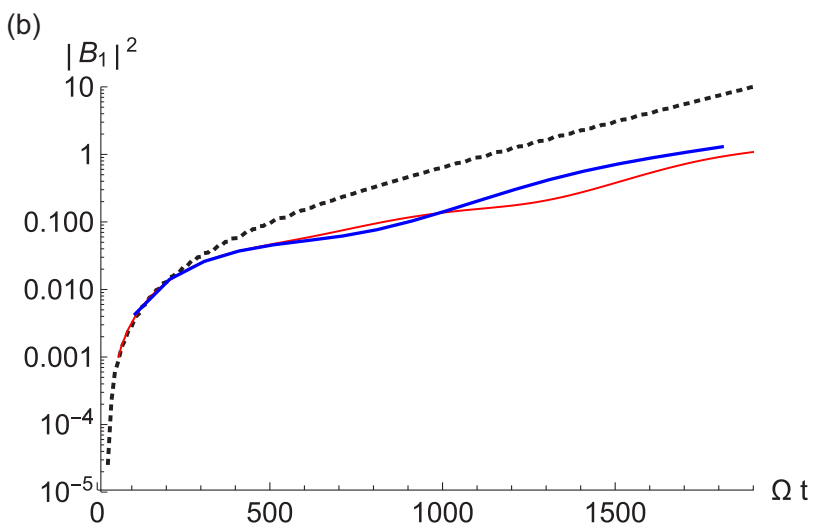

FIG. 1. (a) Behavior of the $\left|B_{1}\right|^{2}$ coefficient as a function of the dimensionless time for different values of $M$ under the perturbation of $\Omega=2 \omega_{1}$. The gray solid (upper) curve represents the analytical solution for the number of particles created when $M=10$, while the blue solid (lower) curve is the numerical solution obtained for the right-moving mirror. (b) Behavior of the $\left|B_{1}\right|^{2}$ coefficient as a function of the dimensionless time for smaller values of $M$, i.e., $M=0.01, M=0.05$, and $M=1$. Parameters used are $\epsilon=0.001, \Lambda=10$. 
(a)

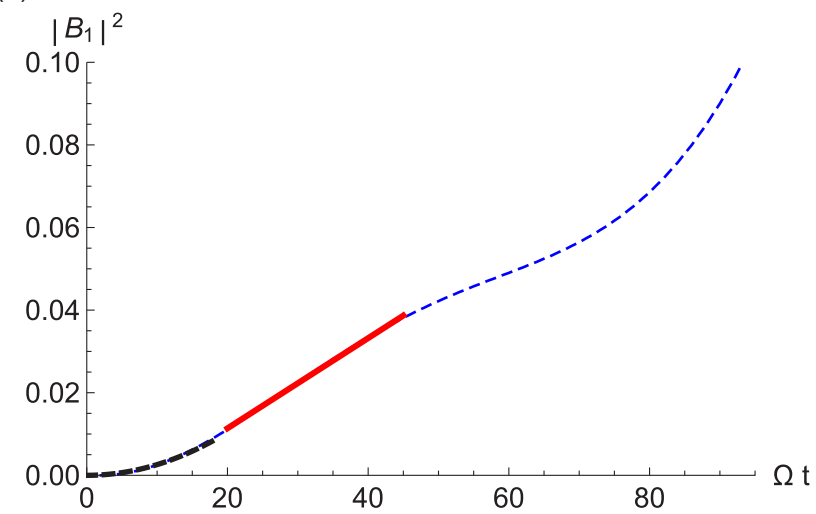

(b)

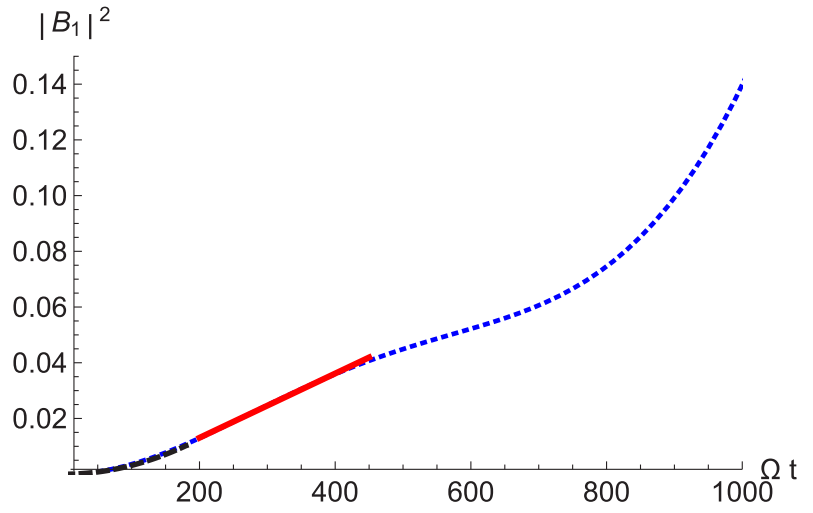

FIG. 2. In both plots, we can see the behavior of the $\left|B_{1}\right|^{2}$ coefficient of the mode $\omega_{1}$ of the field as a function of the dimensionless time. For times smaller than $1 / \epsilon$, the behavior can be fitted by a quadratic curve, while for times $\Omega t \sim 1 / \epsilon$ it can be fitted with a linear-in-time curve. For very long times, we find an exponential behavior, as shown in Ref. [31]. (a) We use $\epsilon_{R}=0.01$ and. (b) We set $\epsilon_{R}=0.001$. Parameters used are $M=0, \Lambda=10$.

containing 10 field modes. The red dashed line represents a 50-mode field cavity, while for the dotted black line, the cavity contains 100 modes. The upper curves represent the $\left|B_{1}\right|^{2}$ coefficient, while the lower ones are for $\left|B_{2}\right|^{2}$. It is evident that at short times, all cavities yield the same results, but at very long times it is wiser to consider a larger number of modes. However, the qualitative behavior remains the same; we obtain lower exponential growth as the number of modes considered increases. In this section we have mainly proved numerically previous results and investigated some regions beyond the analytical studies. In the following section we will analyze the more general case of two moving mirrors.

\section{TWO MOVING MIRRORS}

Reference [29] presented a unified analytic treatment of the dynamical Casimir effect in a one-dimensional resonantly oscillating cavity for arbitrary amplitude and dephasing. Therein, it was shown that for certain cases there is destructive

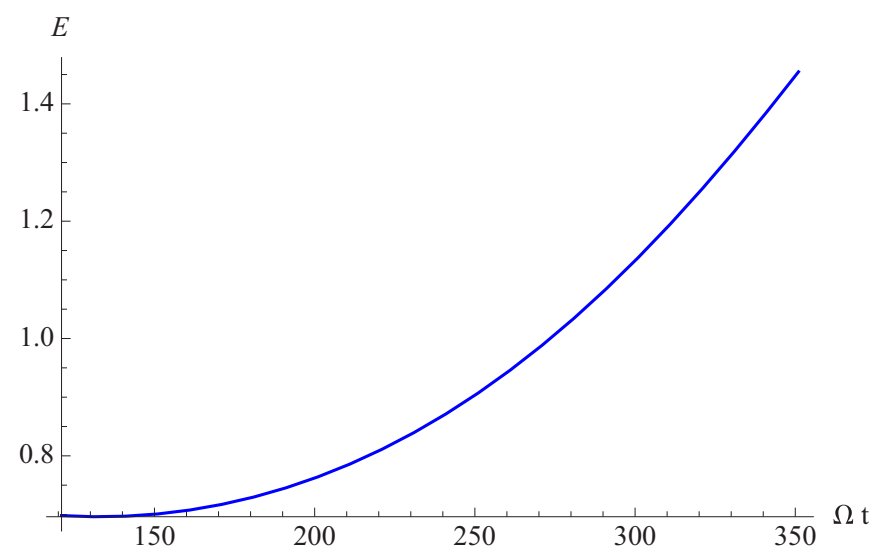

FIG. 3. Energy density for a moving wall located at $x=R(t)$ as a function of the dimensionless time. The density of energy grows quadratically with time in the one-dimensional case of one moving mirror. Parameters are $\epsilon=0.001, \Lambda=10$. In our units, energy is measured in units of $1 / L_{0}$. interference and no radiation is generated. For others, there is constructive interference, and motion-induced photons appear. When this takes place, the way the energy and number of created photons inside the cavity grow in time depends on the relation among several parameters. For certain motions the growth of the energy density is exponential, and for some others it is a power law. In this section, we shall simulate the photon creation of two moving walls for different situations. We will assume the case where $M \ll(n \pi) /(R-L)$ in order to reobtain the results presented in Ref. [29], i.e., a one-dimensional cavity. We will also consider the threedimensional case by including a mass term in the frequency of the field in a nonperturbative treatment.

\section{Non-particle-induced modes}

For a particular case of equal amplitudes $\epsilon_{R}=\epsilon_{L}$ and excitation frequency $\Omega_{R}=\Omega_{L}=\omega_{n}$, where $n$ indicates the

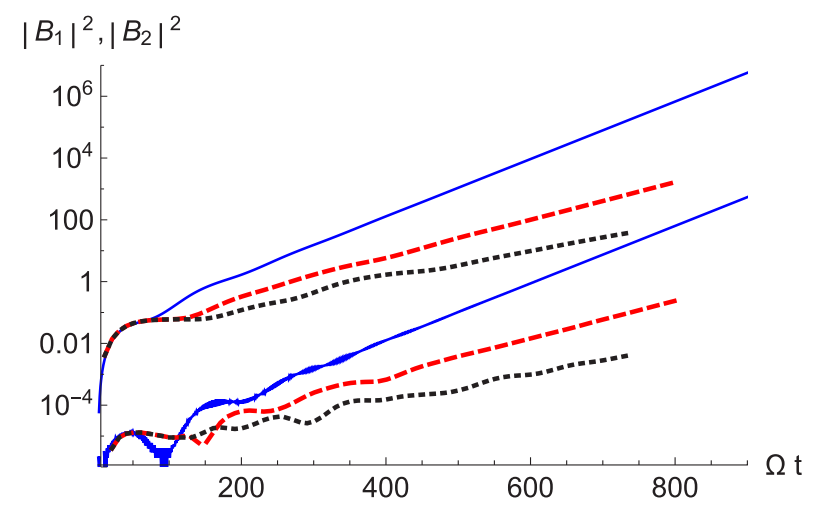

FIG. 4. In this plot, we have used $\epsilon=0.01, \Lambda=10$ for the blue solid curves, $\Lambda=50$ for the red dashed curves, and $\Lambda=100$ for the black dotted ones. In the upper values of the $|B|^{2}$ axis we plot $\left|B_{1}\right|^{2}$ for the $n=1$ mode of the field, while in the lower part of the axis we show $\left|B_{2}\right|^{2}$ of the field as a function of the dimensionless time. We need to use a large number of modes $\Lambda$ in order to get total convergence into the final values. This plots show the dependence on $\Lambda$ and a tendency to exponential growth of created particles. 

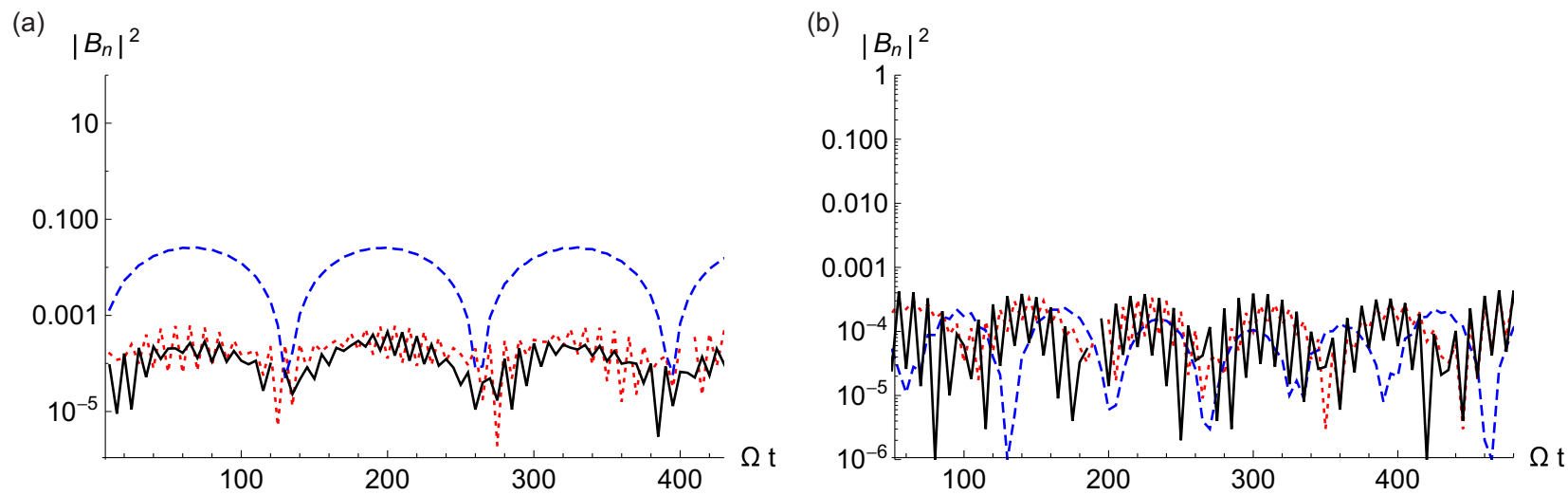

FIG. 5. (a) $|B|^{2}$ coefficient as a function of the dimensionless time for different field modes: $\left|B_{3}\right|^{2}$ (red dotted line), $\left|B_{5}\right|^{2}$ (blue dashed line), and $\left|B_{7}\right|^{2}$ (blue solid line) under $\Omega_{R}=\Omega_{L}=\omega_{5}$ and $\phi_{R}=\pi$. (b) $|B|^{2}$ coefficient for different field modes: $\left|B_{3}\right|^{2},\left|B_{4}\right|^{2}$, and $\left|B_{5}\right|^{2}$ for $\Omega_{R}=\Omega_{L}=\omega_{4}$ and $\phi_{R}=0$. Parameters used are $\phi_{L}=0, \epsilon=0.01, \Lambda=10$.

mode field, the energy inside the cavity oscillates around the static Casimir value, and there is no motion-induced radiation. This was first reported in [28] for a perturbative treatment. It can be shown that for an even value of $n$ and $\phi_{R}=0$ or an odd value of $n$ and $\phi_{R}=\pi$, there is destructive interference among the two moving mirrors [29] (assuming $\phi_{L}=0$ in all cases). This particular behavior is represented in Figs. 5(a) and 5(b), where we show destructive interference and no creation of particles.

\section{Dephased mirrors}

In this section we follow the study of the cavity with two moving mirrors, analyzing the case where the mirrors move in dephased trajectories. In particular, in Fig. 6 we show different cases in which particle creation for odd modes depends on the different values of the phase $\phi_{R}$ for a fixed value of $\phi_{L}=0$.

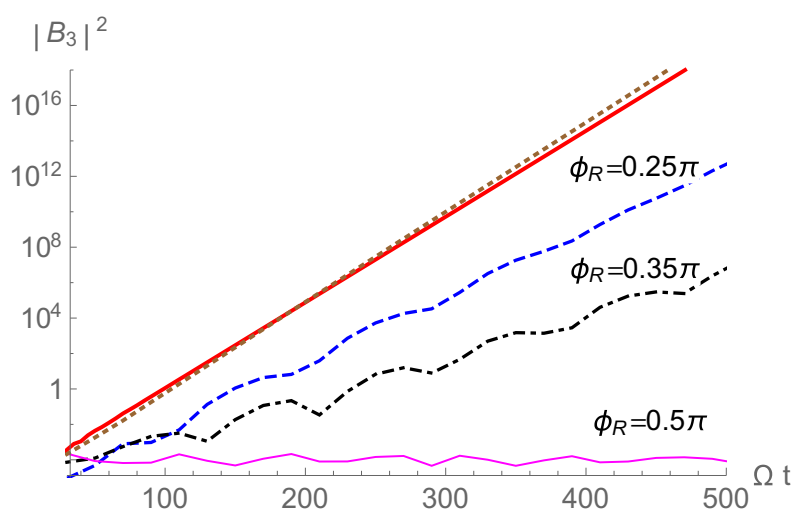

FIG. 6. We present the behavior of an odd field mode $\left|B_{3}\right|^{2}$ as a function of the dimensionless time for different values of $\phi_{R}$ under a perturbation $\Omega=\omega_{5}$. In the case of $\phi_{L}=\phi_{R}=0$ (dotted gray line), we see there is another slope (solid red line) which corresponds to a dephasing of $\phi_{L}=\phi_{R}=\pi / 4$. Both cases are examples of a translational oscillation mode, a case which is considered separately. With the blue dashed line we present $\phi_{L}=0$ and $\phi_{R}=\pi / 4$, and we show $\phi_{L}=0$ and $\phi_{R}=0.35 \pi$ with the dot-dashed black line. Both examples show exponential growth with time of the number of particles created with excitation $\Omega=w_{5}$ for mode $n=3$. Parameters used are $\epsilon=0.01, \Lambda=10$.
In this general case, the number of created particles grows exponentially with time for dephased motion and oscillates nonexponentially for the particular case of $\phi_{L}=0$ and $\phi_{R}=$ $\pi / 2$ (solid pink line in Fig. 6) when the excitation is $\Omega=$ $\omega_{5}$. This case is in agreement with the result obtained in the previous section since it is equivalent to the total destructive interference example where there is no particle creation. We have also included cases of trajectories in phase (translational oscillation mode) as $\phi_{L}=0$ and $\phi_{R}=0$ (dotted gray line in Fig. 6) and $\phi_{L}=\phi_{R}=\pi / 4$ (solid red line in Fig. 6) in which the behavior is also exponential, as we will see in the following section. Furthermore, with the dashed blue line we show $\phi_{L}=0$ and $\phi_{R}=\pi / 4$, and we show $\phi_{L}=0$ and $\phi_{R}=$ $0.35 \pi$ with the dot-dashed black line. Both examples show an exponential growth in time of the number of particles created with excitation $\Omega=\omega_{5}$ for mode $n=3$.

\section{Translational modes}

For the particular situation where $\epsilon_{R}=\epsilon_{L}=\epsilon, \Omega_{R}=\Omega_{L}$, and $\phi_{L}=\phi_{R}=0$, the cavity oscillates as a whole. In this case, the mechanical length is kept constant and is pictorially called an "electromagnetic shaker" [29]. As in the previous examples, it is well known that, due to parametric resonance, a naive perturbative solution of Eq. (6), in powers of $\epsilon$, breaks down after a short amount of time. In order to find an analytical solution valid for longer times one can use the multiple-scale analysis (MSA) technique [11,32]. The MSA provides us with a simple technique equivalent to summing the most secular terms to all orders in the perturbative treatment. In this way, it is possible to get a solution valid for a period of time longer than the perturbative case. We shall introduce a second time scale $\tau=\epsilon t$ and write Eq. (9) as

$$
Q_{n}(t, \tau)=\frac{A_{n}(\tau)}{\sqrt{2 \omega_{n}}} e^{-i \omega_{n} t}+\frac{B_{n}(\tau)}{\sqrt{2 \omega_{n}}} e^{i \omega_{n} t},
$$

where the functions $A_{n}$ and $B_{n}$ are slowly varying and contain the cumulative resonant effects. To obtain differential equations for them, we insert this ansatz into Eq. (6), expand in powers of $\epsilon$ up to first order, and neglect second derivatives of $A_{n}$ and $B_{n}$. The basic idea of MSA is to impose the condition that any term on the right-hand side of the previous equation 
(a)

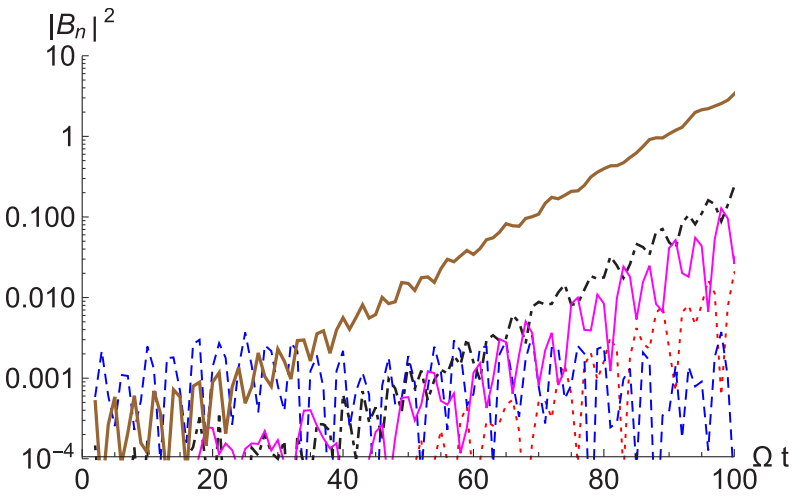

(b)

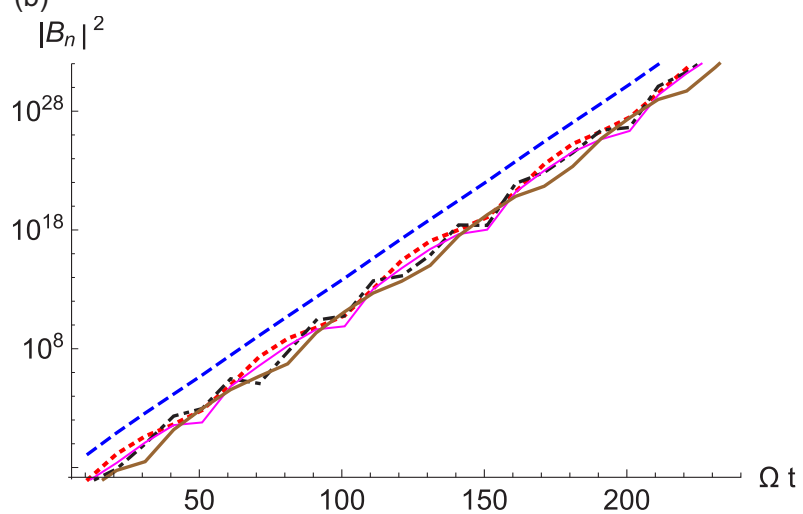

FIG. 7. (a) Solid lines are for $\left|B_{8}\right|^{2}$ and $\left|B_{5}\right|^{2}$; the others lines correspond to $\left|B_{3}\right|^{2},\left|B_{2}\right|^{2}$, and $\left|B_{1}\right|^{2}$. Parameters used are $\epsilon=0.01, \Lambda=10$, $M=10$. (b) All modes are parametrically excited for a bigger value of the mass. Parameters used are $\epsilon=0.001, \Lambda=10, M=50$. Excitation frequency $\Omega=\omega_{2}+\omega_{3}$.

with a time dependency of the form $e^{ \pm i \omega_{n} t}$ must vanish. If not, these terms would be in resonance with the left-hand-side term, and secularities would appear. We will follow this procedure in order to get some analytical predictions about particle creation in the translational mode. After imposing the requirement that no term $e^{ \pm i \omega_{n} t}$ will appear, we get

$$
\begin{aligned}
\frac{d A_{n}}{d \tau}= & \frac{A_{0} \Omega}{4 L_{0} \omega_{n}} \sum_{m} \omega_{m} \tilde{b}_{n m}^{(1)}\left\{\left[\delta\left(\Omega+\omega_{m}-\omega_{n}\right)+\delta\left(\Omega-\omega_{m}+\omega_{n}\right)\right] A_{m}-\left[\delta\left(\Omega-\omega_{m}-\omega_{n}\right)+\delta\left(\Omega+\omega_{m}+\omega_{n}\right)\right] B_{m}\right\} \\
& -\frac{A}{16 L_{0}^{2} \omega_{n}} \sum_{m} \tilde{g}_{n m}^{(1)}\left\{\left[\delta\left(\Omega+\omega_{m}-\omega_{n}\right)-\delta\left(\Omega-\omega_{m}+\omega_{n}\right)\right] A_{m}+\left[\delta\left(\Omega-\omega_{m}-\omega_{n}\right)-\delta\left(\Omega+\omega_{m}+\omega_{n}\right)\right] B_{m}\right\}, \\
\frac{d B_{n}}{d \tau}= & -\frac{A_{0} \Omega}{4 L_{0} \omega_{n}} \sum_{m} \omega_{m} \tilde{b}_{n m}^{(1)}\left\{\left[\delta\left(\Omega+\omega_{m}+\omega_{n}\right)+\delta\left(\Omega-\omega_{m}-\omega_{n}\right)\right] A_{m}-\left[\delta\left(\Omega-\omega_{m}+\omega_{n}\right)+\delta\left(\Omega+\omega_{m}-\omega_{n}\right)\right] B_{m}\right\} \\
& +\frac{A}{16 L_{0}^{2} \omega_{n}} \sum_{m} \tilde{g}_{n m}^{(1)}\left\{\left[\delta\left(\Omega+\omega_{m}+\omega_{n}\right)-\delta\left(\Omega-\omega_{m}-\omega_{n}\right)\right] A_{m}+\left[\delta\left(\Omega-\omega_{m}+\omega_{n}\right)-\delta\left(\Omega+\omega_{m}-\omega_{n}\right)\right] B_{m}\right\},
\end{aligned}
$$

where $A_{0}$ is the amplitude of the mirror displacement and the first-order coefficients are given by

$$
\tilde{b}_{n m}^{(1)}=\frac{4 n m}{n^{2}-m^{2}}\left[(-1)^{n+m}-1\right], \quad \tilde{g}_{n m}^{(1)}=-\frac{n m}{n^{2}-m^{2}} 8 L_{0} \Omega^{2}\left[(-1)^{n+m}-1\right] .
$$

In these equations, there is not a time-dependent frequency term from which one can get one resonant mode evolution when exciting the system with $\Omega=2 \omega_{n}$. Therefore, in this case, the perturbation part of the mode equation is given in terms of an infinite sum of resonant terms. It is easy to see that, in the small amplitude of the perturbative regime, we will obtain an exponential-in-time number of created particles when $\Omega=\left|\omega_{n} \pm \omega_{m}\right|$ [with $n+m$ being an odd number from Eq. (20)]. Therefore, only those modes $n$ and $m$ will be parametrically excited [11,31], and other modes will not be excited. In order to set a simple example, we can suppose that the external frequency is given by $\Omega=\omega_{2}+\omega_{3}$. In this case, the linear equations are given by

$$
\begin{aligned}
& \ddot{A}_{2}-\left(\frac{6 A_{0} \Omega}{5 L_{0}}\right)^{2} \frac{\left(\omega_{3}-\omega_{2}\right)^{2}}{\omega_{2} \omega_{3}} A_{2}=0, \\
& \ddot{B}_{2}-\left(\frac{6 A_{0} \Omega}{5 L_{0}}\right)^{2} \frac{\left(\omega_{3}-\omega_{2}\right)^{2}}{\omega_{2} \omega_{3}} B_{2}=0,
\end{aligned}
$$

and the solution for the $B_{2}$ coefficient is $B_{2}(t) \sim \exp (\Gamma \epsilon t)$, where the rate $\Gamma=\left(6 A_{0} / 5 L_{0}\right)\left(\omega_{3}^{2}-\omega_{2}^{2}\right) / \sqrt{\omega_{2} \omega_{3}}$. This will make $N_{2}$ be exponential with time.

The same conclusion could be obtained for the mode $n=$ 3 , and that would make $N_{3}$ also an exponential function of time. All other modes different from $n=2$ and $n=3$ are not parametrically excited in the perturbative regime under MSA.

However, for given values of $\epsilon$ and $M$, the perturbation in the Eqs. (18) and (19) is large, and the perturbative and MSA approaches are no longer valid. Nonetheless, our numerical evidence goes beyond the perturbative regime and the MSA improvement itself, and we can study the mode fields at longer times. In Fig. 7, we show an example of a nonperturbative result in which we find an exponentially growing number of created particles for most (or all) modes after exciting the system with $\Omega=\omega_{2}+\omega_{3}$. This is, in principle, a result not expected from the perturbative approach mentioned above. In Fig. 7(a), we can see the behavior of some field modes for the excitation $\Omega=\omega_{2}+\omega_{3}$, where some modes oscillate while other are already excited for time $\Omega t \sim 1 / \epsilon$. In Fig. 7(b), for a larger mass, we can see that all modes are 
(a)

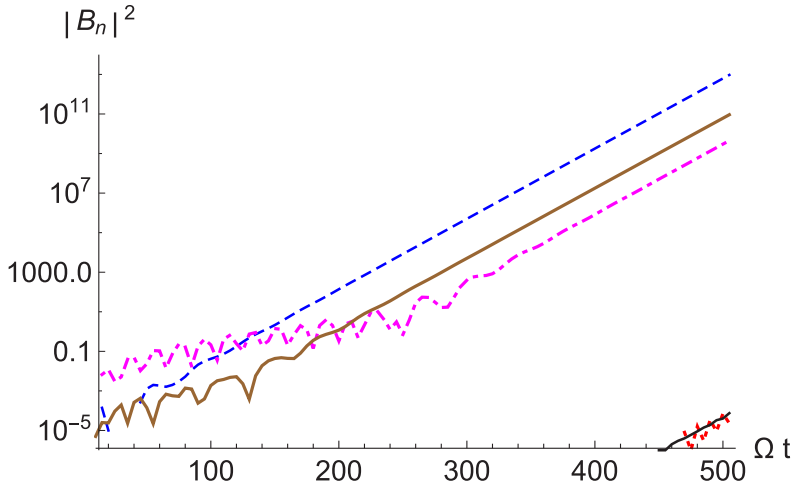

(b)

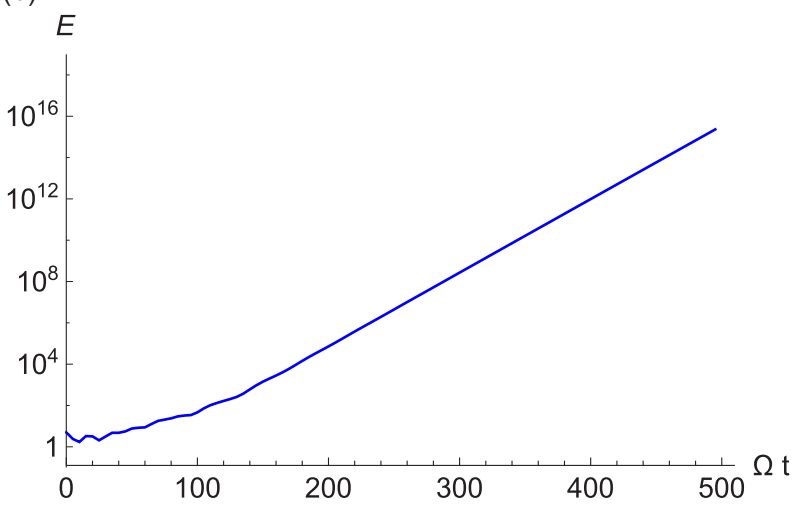

FIG. 8. (a) For the breathing modes, we have the $|B|^{2}$ coefficient versus time for different field modes $n=2\left(\left|B_{2}\right|^{2}\right), n=4\left(\left|B_{4}\right|^{2}\right)$, and $n=6\left(\left|B_{6}\right|^{2}\right)$ under $\Omega_{R}=\Omega_{L}=\omega_{4}$ and $\phi_{L}=\pi$. Field modes $n=1\left(\left|B_{1}\right|^{2}\right)$ and $n=3\left(\left|B_{3}\right|^{2}\right)$ seem to get excited at longer times. (b) Energy density as a function of the dimensionless time. Parameters used are $\epsilon=0.01, \Lambda=10$. In our units, energy is measured in units of $1 / L_{0}$.

parametrically excited for the same time scale, even though $\epsilon$ is smaller.

\section{Breathing modes}

We next consider the case in which $\epsilon_{R}=\epsilon_{L}, \Omega_{R}=\Omega_{L}=$ $\omega_{n}$, and $\phi_{R}=\pi\left(\phi_{L}=0\right)$, representing the situation in which the mirrors oscillate symmetrically with respect to the center of the cavity. The mechanical length changes periodically as an "antishaker" in relation to the previous example in which the cavity moves as a whole. This is simulated in Fig. 8. The dashed blue line shows the mode $n=2$, the dot-dashed magenta line shows $n=4$, and the solid line is for $n=6$. The odd modes, such as $n=1$ and $n=3$, activate at longer times, as shown in Fig. 8(a). In Fig. 8, we excite the system with $\Omega_{R}=\Omega_{L}=\omega_{4}$ and $\phi_{L}=\pi$ with a negligible mass, yielding $\omega_{4}=4 \pi / L_{0}$. Therein, we can observe that all even modes are getting excited, while the odd modes become excited at longer times (they appear at times $\Omega t \sim 500$ ). In the end, all modes become exponentially excited in a nonperturbative example.

\section{Three-dimensional cavities with two moving mirrors}

We can even study particle creation in the three-dimensional cavity with two moving mirrors. We start increasing the value

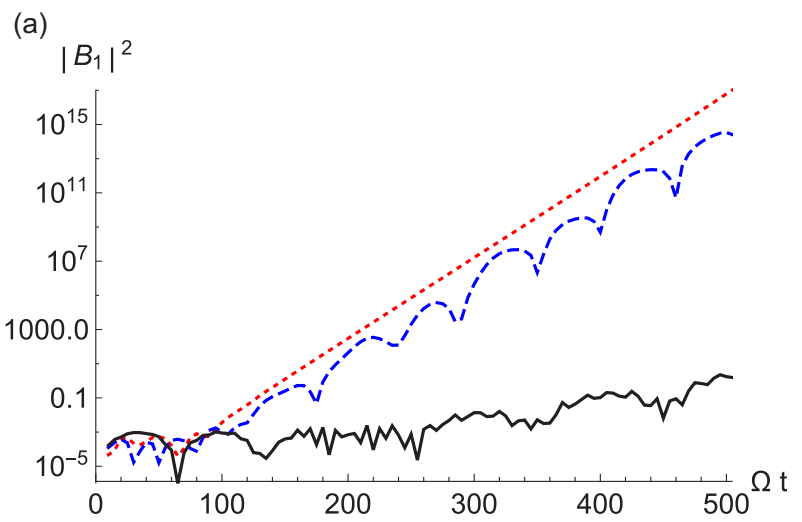

of the mass and see how the explosive cocktail of Fig. 9 starts changing considerably. In Fig. 9, we show the behavior of the coefficient for field mode $n=1,\left|B_{1}\right|^{2}$ under a perturbation defined by $\Omega_{R}=\Omega_{L}=\omega_{5}$, and $\phi_{L}=0$ for different values of the mass value: $M=0.01$ (dotted line), $M=1$ (dashed line), and $M=5$ (solid line). In Fig. 9(a), we show the energy as a function of time for the same values of the parameters. Therein, we see that for $M=0.01$, the energy has an exponential behavior, while for $M=5$ it does not.

It is easy to see in Fig. 9 the different results for the number of created particles between one- and three-dimensional cavities. In fact, it is important to note in Fig. 9(a) that the smaller the mass value is, the bigger the growth of the coefficient $\left|B_{1}\right|^{2}$ is. Then, one-dimensional cavities with two oscillating mirrors produce a bigger excitation of modes than the corresponding cubic cavity in three dimensions. The same mass hierarchy can be seen in Fig. 9(b) for the energy density inside the cavities (one-dimensional cavity when $M \rightarrow 0$ and three-dimensional ones for bigger $M$ ).

\section{CONCLUSIONS}

In this paper we presented a detailed numerical analysis of particle creation for a quantum field in a cavity with

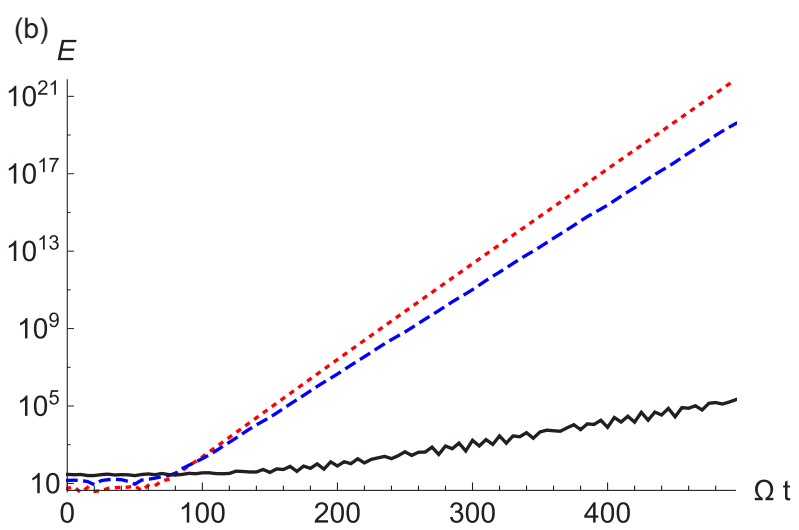

FIG. 9. (a) $B_{1}$ coefficient for field mode 1 under $\Omega_{R}=\Omega_{L}=\omega_{5}$ and $\phi_{L}=0$ for different values of the mass: $M=0.01$ (red dotted line), $M=1$ (blue dashed line), and $M=5$ (black solid curve). (b) Energy as a function of time. Parameters used are $\epsilon=0.01, \Lambda=10$. In our units, energy is measured in units of $1 / L_{0}$. 
two perfectly conducting moving mirrors. This approach was applied to one-dimensional and three-dimensional cavity boxes. We derived the equation of motion of the field modes and numerically evaluated the Bogoliubov transformation between in and out states. From Bogoliubov coefficients, we were able to numerically calculate the number of created particles after the mirrors stop moving and return to the unperturbed position.

In the case of one moving wall at $x=R(t)$ while the wall at $x=0$ remains at rest, we recovered the very well known results. In that case, we showed that the rate of particle production depends strongly on whether the frequency spectrum is equidistant or not, obtaining the correct behavior with respect to the number of created particles. Quadratic behavior for a short time scale and linear growth for larger temporal scales were found when the spectrum was equidistant. We also found that, beyond perturbative predictions, the dependence on the number of created particles is exponential with time for very large times. In the case of three-dimensional cavities with one moving mirror, we showed that our results are in agreement with the literature for the perturbative or MSA regions and provided numerical evidence of the behavior of the number of created particles even in the nonperturbative case.

In the case of two moving walls, we showed that the rate of particle creation depends strongly on the relation among the amplitudes, the frequency, and the phase difference in the mirrors' oscillations. We showed that in some cases constructive interference leads to exponential growth of particles inside the cavity, while for other relations there exists destructive interference with no vacuum radiation. We went beyond analytical studies by considering a massive field and computing the energy density inside the cavity.

We also studied nonperturbative regimes for translational modes and found that all modes in the cavity grow exponentially when the amplitude of the perturbation in the mode equation is large (compared with perturbative expansion analysis). Then, we reported an exponentially growing number of created particles when exciting with external frequencies $\Omega=\left|\omega_{n} \pm \omega_{m}\right|$ (with $n+m$ being an odd number). We will present further analysis of the translational mode oscillation with analytical and more numerical support elsewhere [33].

\section{ACKNOWLEDGMENTS}

This work was supported by ANPCyT, CONICET, and Universidad de Buenos Aires (UBA). We thanks F. D. Mazzitelli for useful comments.
[1] D. A. R. Dalvit, P. A. Maia Neto, and F. D. Mazzitelli, in Casimir Physics, Lecture Notes in Physics Vol. 834, edited by D. Dalvit, P. Milonni, D. Roberts, and F. da Rosa (Springer, Berlin, 2011), p. 419.

[2] V. V. Dodonov, Adv. Chem. Phys. 119, 309 (2001).

[3] V. V. Dodonov, Phys. Scr. 82, 038105 (2010).

[4] P. D. Nation, J. R. Johansson, M. P. Blencowe, and F. Nori, Rev. Mod. Phys. 84, 1 (2012).

[5] C. K. Law, Phys. Rev. A 49, 433 (1994).

[6] S. A. Fulling and C. W. Davies, Proc. R. Soc. London, Ser. A 348, 393 (1976).

[7] V. V. Dodonov and A. B. Klimov, Phys. Rev. A 53, 2664 (1996).

[8] D. F. Mundarain and P. A. Maia Neto, Phys. Rev. A 57, 1379 (1998).

[9] V. V. Dodonov, Phys. Lett. A 244, 517 (1998).

[10] M. Ruser, J. Phys. A 39, 6711 (2006); D. T. Alves and E. R. Granhen, Comput. Phys. Commun. 185, 2101 (2014).

[11] M. Crocce, D. A. R. Dalvit, and F. D. Mazzitelli, Phys. Rev. A 64, 013808 (2001).

[12] A. V. Dodonov and V. V. Dodonov, Phys. Lett. A 289, 291 (2001).

[13] C. M. Wilson, G. Johansson, A. Pourkabirian, M. Simoen, J. R. Johansson, T. Duty, F. Nori, and P. Delsing, Nature (London) 479, 376 (2011).

[14] P. Lhteenmaki, G. S. Paraoanu, J. Hassel, and P. J. Hakonen, Proc. Natl. Acad. Sci. USA 110, 4234 (2013).

[15] S. Felicetti, C. Sabín, I. Fuentes, L. Lamata, G. Romero, and E. Solano, Phys. Rev. B 92, 064501 (2015).

[16] G. Benenti, A. D’Arrigo, S. Siccardi, and G. Strini, Phys. Rev. A 90, 052313 (2014).
[17] J. R. Johansson, G. Johansson, C. M. Wilson, P. Delsing, and F. Nori, Phys. Rev. A 87, 043804 (2013).

[18] S. Felicetti, M. Sanz, L. Lamata, G. Romero, G. Johansson, P. Delsing, and E. Solano, Phys. Rev. Lett. 113, 093602 (2014).

[19] R. Stassi, S. De Liberato, L. Garziano, B. Spagnolo, and S. Savasta, Phys. Rev. A 92, 013830 (2015).

[20] A. Agnesi, C. Braggio, G. Bressi, G. Carugno, G. Galeazzi, F. Pirzio, G. Reali, G. Ruoso, and D. Zanello, J. Phys. A: Math. Theor. 41, 164024 (2008). For a model with time dependent conductivity, see M. Crocce, D. A. R. Dalvit, F. C. Lombardo, and F. D. Mazzitelli, Phys. Rev. A 70, 033811 (2004).

[21] W.-J. Kim, J. H. Brownell, and R. Onofrio, Phys. Rev. Lett. 96, 200402 (2006).

[22] A. V. Dodonov, J. Phys. Conf. Ser. 161, 012029 (2009).

[23] S. De Liberato, D. Gerace, I. Carusotto, and C. Ciuti, Phys. Rev. A 80, 053810 (2009).

[24] A. V. Dodonov, J. Phys. A 47, 285303 (2014).

[25] I. M. de Sousa and A. V. Dodonov, J. Phys. A 48, 245302 (2015).

[26] D. S. Veloso and A. V. Dodonov, J. Phys. B 48, 165503 (2015).

[27] G. Vacanti, S. Pugnetti, N. Didier, M. Paternostro, G. M. Palma, R. Fazio, and V. Vedral, Phys. Rev. Lett. 108, 093603 (2012).

[28] J.-Y. Ji, H.-H. Jung, and K.-S. Soh, Phys. Rev. A 57, 4952 (1998).

[29] D. A. R. Dalvit and F. D. Mazzitelli, Phys. Rev. A 59, 3049 (1999). 
[30] For the case of a $3+1$ field, the procedure is similar by assuming the instantaneous mode basis as

$$
\begin{aligned}
\phi_{k}(\vec{x}, t)= & \sqrt{\frac{2}{R-L}} \sin \left(\frac{k_{x} \pi(x-L)}{R-L}\right) \sqrt{\frac{2}{L_{y}}} \\
& \times \sin \left(\frac{k_{y} \pi y}{L_{y}}\right) \sqrt{\frac{2}{L_{z}}} \sin \left(\frac{k_{z} \pi z}{L_{z}}\right) .
\end{aligned}
$$

[31] F. C. Lombardo, F. D. Mazzitelli, A. Soba, and P. I. Villar, Phys. Rev. A 93, 032501 (2016).

[32] C. M. Bender and S. A. Orszag, Advanced Mathematical Methods for Scientists and Engineers (McGraw-Hill, New York, 1978).

[33] F. C. Lombardo, F. D. Mazzitelli, A. Soba, and P. I. Villar (unpublished). 